\title{
Evaluation of measurement properties of self-administered PROMs aimed at patients with non-specific shoulder pain and "activity limitations": a systematic review
}

\author{
M. Thoomes-de Graaf ${ }^{1,2}$ - G. G. M. Scholten-Peeters ${ }^{1,2,3} \cdot$ J. M. Schellingerhout ${ }^{4}$ • \\ A. M. Bourne ${ }^{6}$ R. Buchbinder ${ }^{5,6} \cdot$ M. Koehorst ${ }^{7}$ C. B. Terwee ${ }^{8}$. \\ A. P. Verhagen ${ }^{1,2}$
}

Accepted: 21 March 2016/Published online: 2 April 2016

(c) The Author(s) 2016. This article is published with open access at Springerlink.com

\begin{abstract}
Objective To critically appraise and compare the measurement properties of self-administered patient-reported outcome measures (PROMs) focussing on the shoulder, assessing "activity limitations."

Study design Systematic review. The study population had to consist of patients with shoulder pain. We excluded postoperative patients or patients with generic diseases. The methodological quality of the selected studies and the results of the measurement properties were critically appraised and rated using the COSMIN checklist.

Results Out of a total of 3427 unique hits, 31 articles, evaluating 7 different questionnaires, were included. The SPADI is
\end{abstract}

M. Thoomes-de Graaf

marloesthoomes@me.com

G. G. M. Scholten-Peeters

ggm.scholten@gmail.com

J. M. Schellingerhout

j.schellingerhout@erasmusmc.nl

A. M. Bourne

allison.bourne@gmail.com

R. Buchbinder

rachelle.buchbinder@monash.edu

M. Koehorst

M.Sleijser-Koehorst@somt.nl

C. B. Terwee

cb.terwee@vumc.nl

A. P. Verhagen

a.verhagen@erasmusmc.nl

1 Research Group Diagnostics, Avans University of Applied Science, Hogeschoollaan 1, 4818 CR Breda, The Netherlands

2 Department of General Practice, Erasmus Medical Centre, Wytemaweg 80, 3015 CN Rotterdam, The Netherlands the most frequently evaluated PROM and its measurement properties seem adequate apart from a lack of information regarding its measurement error and content validity.

Conclusion For English, Norwegian and Turkish users, we recommend to use the SPADI. Dutch users could use either the SDQ or the SST. In German, we recommend the DASH. In Tamil, Slovene, Spanish and the Danish languages, the evaluated PROMs were not yet of acceptable validity. None of these PROMs showed strong positive evidence for all measurement properties. We propose to develop a new shoulder PROM focused on activity limitations, taking new knowledge and techniques into account.

3 Faculty of Behavioural and Movement Sciences, MOVE Research Institute Amsterdam, VU University Amsterdam, Van der Boechorststraat 9, 1081 BT Amsterdam, The Netherlands

4 Het Huisartsenteam De Keen, Voorsteven 88, 4871 DX Etten-Leur, The Netherlands

5 Monash Department of Clinical Epidemiology, Cabrini Institute, Suite 41, 183 Wattletree Rd, Malvern, Melbourne, VIC 3144, Australia

6 Department of Epidemiology and Preventive Medicine, The Alfred Centre, School of Public Health and Preventive Medicine, Monash University, 99 Commercial Road, Melbourne, VIC 3004, Australia

7 SOMT Institute for Master Education in Musculoskeletal Therapies, Softwareweg 5, 3821 BN Amersfoort, The Netherlands

8 Department of Epidemiology and Biostatistics and the EMGO Institute for Health and Care Research, VU University Medical Center, Van der Boechorststraat 7, 1081 BT Amsterdam, The Netherlands 
Keywords Shoulder pain · Disability - Questionnaire . Patient outcome assessment · Psychometrics · Systematic review

\section{Introduction}

The International Classification of Functioning, Disability and Health (ICF) have described the widely accepted definition of functional health status in terms of "impairments," "activity limitations," and "participation restrictions" [1-3]. For patients with shoulder pain, one of the most important consequences in terms of their health is "activity limitations" [4]. As such, health-related patientreported outcome measures (PROMs) that assess perceived "activity limitations" are useful in terms of assessing the physical impairment in patients with shoulder pain.

Several PROMs focusing on the shoulder have been developed to measure "activity limitations" in patients with shoulder pain. Examples of these include the Shoulder Disability Questionnaire (SDQ) [5] and the Shoulder Pain and Disability Index (SPADI) [6]. Furthermore, the disabilities of the arm, shoulder and hand questionnaire (DASH) is also often used for patients with shoulder pain [7]. There is a great variety in PROMs focusing on patients with shoulder pain. Some PROMs, such as the American Shoulder and Elbow Surgeon questionnaire (ASES), include a physical examination component, while others are completely self-administered. Other PROMs are specifically designed for a subgroup of patients, such as the wheelchair user's shoulder pain index (WUSPI), which is specifically designed for wheelchair users.

Several systematic reviews have evaluated the measurement properties of shoulder-specific PROMS. A systematic review which included studies until 2002 found that none of the included 16 PROMs demonstrated satisfactory results for all measurement properties, but overall, the DASH received the best ratings [8]. Another review that assessed the measurement properties of four commonly used shoulder PROMs concluded that none of the questionnaires was superior or could be recommended over the other [9]. A recent review, specifically focused on patients with rotator cuff disorders (RCD), evaluated 12 PROMs and concluded that the included questionnaires showed acceptable psychometric properties for individuals with RCD [10]. Several other reviews have summarized the characteristics and measurement properties of a limited number of PROMs, but these reviews did not assess the methodological quality of the included studies and consequently their conclusions have several limitations [11-13].

Despite the fact that several reviews have been performed, we feel there is a need for a more specific and focused research question. If a research question is broad, it can be difficult to reach conclusions applicable to any single population. For example, a specific description of the patient population is important as it can influence the possibility to reach conclusions [14].

All of the above reviews included studies with mixed populations as well, such as upper extremity disorders. Their recommendations, about PROMs that can be used for patients with shoulder pain explicitly, are partly based on mixed populations, such as patients with solely hand or elbow pain (without shoulder pain). We feel that results of research on psychometric properties of shoulder PROMs should be based on data from patients with shoulder pain only, or should be presented separately. Study populations often consist of patients with "nonspecific" shoulder pain (including rotator cuff disease, frozen shoulder, etc.), but can also include patients with serious pathology (e.g., malignancy, infection and fracture), specific diseases (e.g., rheumatoid arthritis) or postsurgery patients. Especially if responsiveness is assessed, this can have consequences on the results. Therefore, we prefer to include only questionnaires assessing shoulder-related disability in patients with non-specific shoulder pain with or without conservative treatment.

Furthermore, these reviews presented their results per PROM and not per language; however, due to differences in cultural context, a translation of the original version does not guarantee similar psychometric properties $[15,16]$. Therefore, the psychometric qualities of translated PROMs should also be evaluated, before they can be used in daily practice or research.

Recently, a new instrument known as the COSMIN checklist has been developed to evaluate the methodological quality of studies investigating the measurement properties of PROMs [17]. This checklist showed a high level of agreement between raters [17, 18]. Since its development, several systematic reviews examined the measurement properties of various PROMS by means of the COSMIN checklist [19-22].

Therefore, the aim of this study was to critically appraise and compare the measurement properties of both the original versions and the translated versions of selfadministered PROMs focusing on the shoulder assessing "activity limitations" for patients with nonspecific shoulder pain, using the COSMIN checklist.

\section{Methods}

\section{Selection criteria}

We included publications concerning the development or validation/evaluation of measurement properties of an original or translated version of a self-administered PROM 
focussing on the shoulder and assessing "activity limitations". Included patients should have nonspecific shoulder pain as a main complaint. As the definition of adhesive capsulitis, subacromial impingement syndrome and RCD is still unclear and there are no generally accepted criteria yet [23], we consider these pathologies as nonspecific shoulder pain and not as a specific subgroup. Studies including patients with serious pathology (e.g., malignancy, infection and fracture), specific diseases (e.g., rheumatoid arthritis) or where surgery was applied were excluded, as well as studies that did not report their results separately for patients with shoulder pain. Questionnaires including physical examination (e.g., ASES) were excluded, as well as questionnaires specifically designed for specific subgroups, such as RCD [e.g., Western Ontorio Rotator Cuff Index (WORC)], instability [e.g., Western Ontorio Shoulder Instability Index (WOSI)], athletes (e.g., Athletic shoulder outcome rating scale) or wheelchair users (e.g., WUSPI). We explicitly did not exclude studies in which patients with RCD, instability, etc., were used, but we chose to exclude all PROMs that were explicitly designed for a specific subgroup of shoulder complaints, as proposed by their developers.

No language restrictions were applied. Abstracts for which full reports were not available were excluded.

\section{Literature search}

Electronic searches included MEDLINE, EMBASE, CINAHL and Cochrane from inception to August 2014. Eligible studies were identified using MeSH (Medline), Thesaurus (EMBASE, CINAHL) and free text words also including specific names of identified PROMs. We used the highly sensitive and precisely published search filter [24] for PubMed searches and used it to build the subsequent search strategies. We have added the MEDLINE search in the "Appendix," the specific search strings for EMBASE, CINAHL and Cochrane are available from the authors on request. Manual searches of review bibliographies and reference lists of primary studies were also undertaken to search for possible studies not captured by the electronic searches.

A research librarian, together with a review author (MTG) performed the electronic search. Two review authors (MTG, GSP) independently selected the studies to be included by first screening the title and abstract and later assessing the full text papers for eligibility. Disagreements were solved by discussion or through arbitration by a third review author (AV). We listed the excluded studies and their bibliographic details with the reason for exclusion.

\section{Methodological quality}

\section{Quality assessment}

Two reviewer authors (MTG and either JS, AB, MK or $\mathrm{CT}$ ) independently performed the assessment of methodological quality, using the COSMIN checklist [17]. Disagreements were solved by discussion or by a third review author (AV). The checklist contains nine boxes, with standards for good methodological quality of studies on nine different measurement properties [17]. The appropriate boxes were selected per study and each item within this box scored on a 4-point rating scale: "poor," "fair," "good" or "excellent" [25]. An overall score for the methodological quality of a study was determined by taking the lowest rate of any items of the box per measurement property. An intraclass coefficient (ICC) was calculated to assess the immediate agreement between both raters on the overall score per box, and an ICC higher than 0.70 was considered good [26, 27].

\section{Measurement properties}

The measurement properties are divided into three domains: reliability, validity and responsiveness. Information on interpretability and feasibility were also extracted from the studies [17].

\section{Interpretability}

Interpretability is defined as: "the degree to which one can assign qualitative meaning-that is, clinical or commonly understood connotations- to an instrument's quantitative scores or changes in scores" [28]. Information about clinically meaningful differences in scores between subgroups, floor and ceiling effects and the minimal important change (MIC) should be provided [17].

\section{Reliability}

Reliability is defined as: "the extent to which scores for patients who have not changed, are the same for repeated measurement under several conditions." [28].

The reliability domain contains three measurement properties: internal consistency, reliability and measurement error [28]. Internal consistency is "the degree of the interrelatedness among the items" of the questionnaire [28] and is measured by Cronbach's alpha or KuderRichardson Formula 20 or by using IRT methods [17, 27]. Reliability is "the proportion of the total variance in the measurements which is because of 'true' differences 
among patients" [28] and is reflected by the Intraclass Correlation Coefficient (ICC) or Cohen's Kappa [17, 27]. The measurement error is "the systematic and random error of a patient's score that is not attributed to true changes in the construct to be measured" [28]. This can be expressed by the standard error of measurement (SEM), the smallest detectable change (SDC) or the limits of agreement (LoA) [17, 27].

\section{Validity}

Validity is defined as: "the degree to which an instrument measures the construct(s) it purports to measure" [28]. The validity domain also contains three measurement properties: content validity, criterion validity and construct validity [28]. Content validity is "the degree to which the content of an instrument is an adequate reflection of the construct to be measured" and includes face validity [28]. The definition of face validity is "the degree to which (the items of) an instrument indeed looks as though they are an adequate reflection of the construct to be measured" [28]. In assessing this, it is important to consider whether all items are relevant to the originally described construct [17]. Criterion validity is "the degree to which the scores of an instrument are an adequate reflection of a "gold standard"” [28]. As PROMs do not have a "gold standard," criterion validity is not appropriate [17]. Construct validity consists of three items:

1. Structural validity is "the degree to which the scores of an instrument are an adequate reflection of the dimensionality of the construct to be measured" [28]. Factor analysis should be used to determine or confirm existing subscales, which are subsequently used in the hypotheses that are being tested [28].

2. Hypotheses testing is "the degree to which the scores of an instrument are consistent with hypotheses (for instance with regard to internal relationships, relationships to scores of other instruments or differences between relevant groups. Based on the assumption that the instrument validly measures the construct to be measured)" [28].

3. Cross-cultural validity is "the degree to which the performance of the items on a translated or culturally adapted instrument is an adequate reflection of the performance of the items of the original version of the instrument" [28].

\section{Responsiveness}

Responsiveness is defined as: "the ability of an instrument to detect changes over time in the construct to be measured" [28]. Responsiveness is considered to be similar to validity; however, while validity refers to the validity of a single score, responsiveness refers to the validity of a change score [17].

\section{Data extraction}

Two review authors independently performed data extraction (MTG and either JS, AB, MK or CB). Disagreements were resolved by discussion or by a third review author (AV). Descriptive data extracted included the characteristics of the study population (e.g., age, gender, type of shoulder pain, language); general characteristics of the instruments (e.g., construct, subscales, number of items); whether the PROM was an original version or a translated version of the questionnaire and feasibility. Although feasibility is not captured within the COSMIN checklist, the practical use of a questionnaire is important to determine usefulness in clinical practice. Feasibility includes the time needed to complete the questionnaire, its comprehensibility and whether or not it is generally accepted in clinical practice.

Besides, result of the measurement properties and of the interpretability was extracted. Only studies that were ranked as being of fair to excellent methodology were rated on their measurement properties, as studies of poor methodology are of limited value [19, 20].

To rate the results of measurement properties, generally accepted criteria were used [27].

\section{Analysis}

To determine the overall quality of the measurement properties of the different questionnaires we combined the different studies per PROM (for each language) by combining their results (ratings), adjusted for the methodological quality (fair, good or excellent) and the consistency of their results. The overall rating for a measurement property was recorded as "positive," "indeterminate" or "negative." Furthermore, we assessed a level of evidence (strong, moderate, limited, conflicting, unknown) using the COSMIN checklist in a similar manner to that proposed by the Cochrane Review Group (see Table 1) [29].

We made recommendations concerning the use of a certain PROM per language, based upon the best evidence synthesis. Ideally, a PROM should have strong positive evidence on all measurement properties; however, if there was moderate evidence, a recommendation was still made. In case multiple PROMs showed similar ratings in a specific language, both were presented. If there were no studies with at least fair methodology, no recommendations were made and if there was only limited evidence, caution was advised. 
Table 1 Levels of evidence for the overall quality of the measurement property

\begin{tabular}{lll}
\hline Level & Rating $^{\mathrm{a}}$ & Criteria $^{\mathrm{b}}$ \\
\hline Strong & +++ OR --- & Consistent findings among multiple studies of good/excellent methodological quality \\
Moderate & ++ OR - & Consistent finding among multiple studies of fair studies or in one study of good methodological quality \\
Limited & + OR - & One study of fair methodological quality \\
Conflicting & $+/-$ & Conflicting findings \\
Unknown & $?$ & Only studies of poor methodological quality \\
No evidence & 0 & No studies available \\
\hline
\end{tabular}

${ }^{a}$ Rating is based on Table 1 per study, where + refers to a positive result and - for a negative result

b The criteria of methodological quality are based on the COSMIN checklist

\section{Results}

The search strategy resulted in a total of 3421 hits. Of these, 161 articles were selected based on their title and abstract. Reference checking resulted in 6 additional studies. Evaluation of the full text articles resulted in exclusion of 136 articles. Finally, 31 articles, evaluating 7 different questionnaires, were included (see Fig. 1).

The characteristics of the included studies are described in Table 2. For some articles, fewer boxes were scored than described by their original authors, as they did not present these results for our target population separately. The agreement between both raters on the methodological overall quality per box was good [ICC two way random agreement $=0.88(95 \%$ CI $0.818-0.915]$. There was no need to discuss disagreement with the third review author. All original versions were developed in English, except the SDQ, which was originally developed in Dutch. The originally described construct and examples of questions of each PROM are described in Table 3 . The methodological quality of the studies is presented in Table 4 for each PROM for each measurement property. The main categories with poor methodology were internal consistency, reliability and crosscultural validity. The comparator instruments that were used for construct hypothesis testing (except studies of poor methodology) are presented in Table 5. The best evidence synthesis of results per language (per PROM) and their accompanying level of evidence are presented in Table 6 .

Below we will describe the results per questionnaire.

\section{Shoulder pain and disability index (SPADI)}

The SPADI was developed to measure pain and disability associated with shoulder pathology. It consists of 13 items, each scored on a $0-10$ numeric rating scale, divided into two subscales: pain (5 items) and disability (8 items). The total score varies between 0 and 100 [6]. It takes approximately $2-3 \mathrm{~min}$ to complete $[30,31]$. The SPADI is considered to be easy to understand by patients [31], and no floor or ceiling effects have been detected $[32,33]$.

\section{Reliability}

Internal consistency There is strong positive evidence for internal consistency within the English SPADI (Cronbach Alpha $=0.85$ for pain and 0.90 for disability) [34]. There is also limited positive evidence for the internal consistency of the Norwegian SPADI (Cronbach Alpha $=0.80$ for pain and 0.87 for disability) [35]. However, there were inconsistent findings on the factor structure of the SPADI; therefore, these results should be interpreted with caution.

Reliability Both the Norwegian and the Turkish versions showed moderate (ICC $=0.85-0.89)[36,37]$ and limited positive evidence (ICC $=0.92$ ) [38], respectively. Studies evaluating other language versions were rated as having poor methodology.

Measurement error Two studies (both Norwegian) were rated as having at least "fair" methodology that evaluated measurement error, one study of fair methodology only reported an SDC (17 points), but no MIC was determined [36]. The other study reported an SDC of 19.7, and the Loa was between -20.9 and 18.5 [37]; the MIC, however, ranged between 15.0 and 31.1 depending on the methods used [33]; the authors therefore concluded that a change of approximately 20 points is necessary for patient perceived important change.

\section{Validity}

Content validity There were no studies evaluating content validity.

Construct structural validity There is moderate evidence that the English SPADI consists of two factors, pain and disability, and all factors are loaded accordingly as originally proposed by Roach [34]. In contrast, there is limited evidence that not all items are loaded on the original factor, but no explained variance was described [39]. Factor analysis of the Norwegian SPADI resulted in limited 


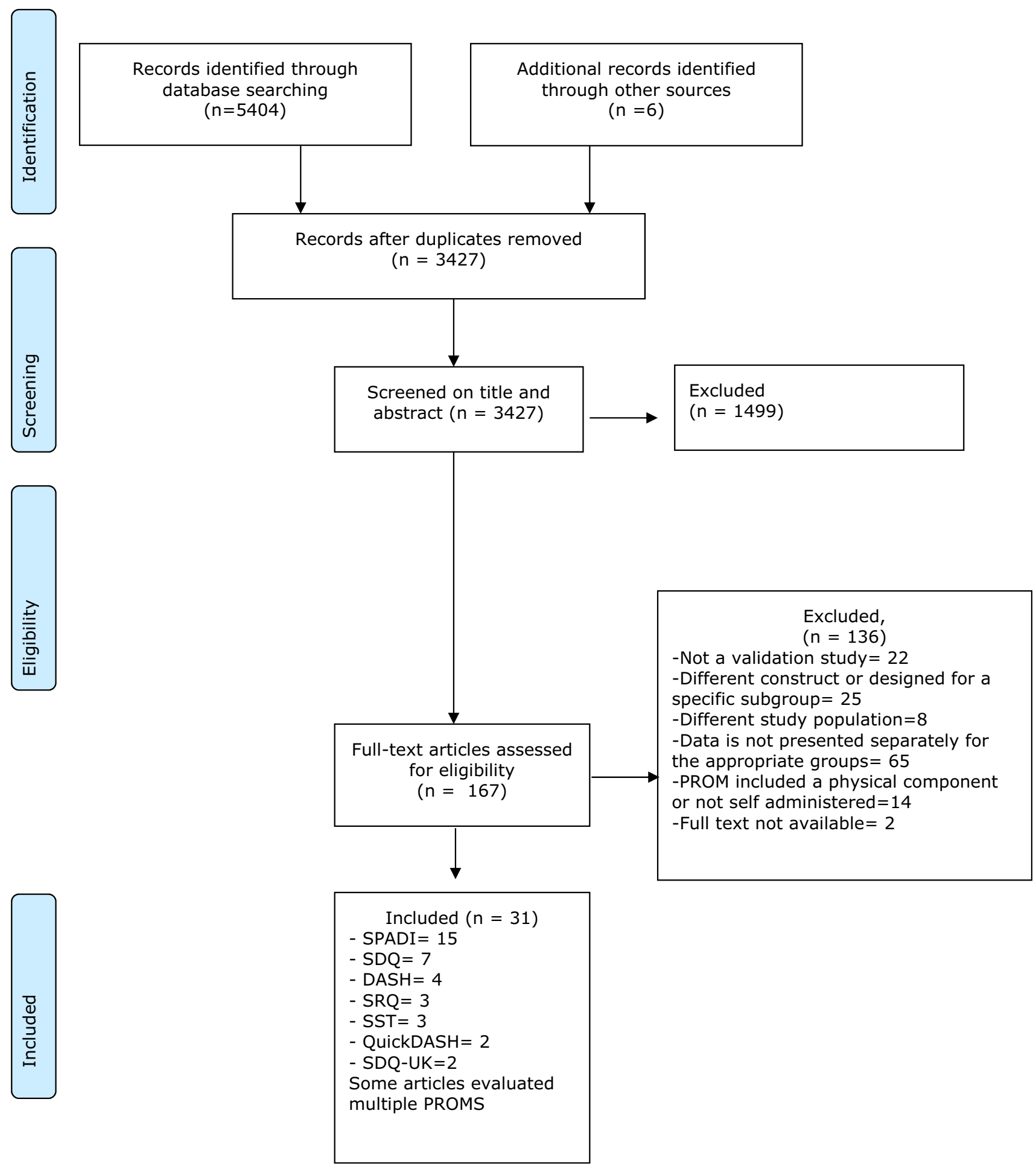

Fig. 1 Inclusion

evidence that it consists of two factors but the original factor structure could not be confirmed, as not all items loaded as originally intended [35].

Construct hypothesis testing In terms of construct hypothesis testing, moderate positive evidence was identified for the English SPADI [31, 39, 40]. There was limited positive evidence for the Turkish version [38] and the Norwegian version [37]. The evidence for the Danish SPADI [32] and the Slovenish version [41] was unclear, as they confirmed their hypothesis with known group validity, but did not assess whether the correlations with 
Table 2 Characteristics of the included studies

\begin{tabular}{|c|c|c|c|c|}
\hline Study & Country & PROMs & Setting & Population \\
\hline \multicolumn{5}{|l|}{ English } \\
\hline Beaton et al. [44] & Canada/USA & DASH & Hospital & $\begin{array}{l}\text { Mixed types of shoulder pain } \\
\text { Mean age } 53,43 \% \text { male }^{\mathrm{a}}\end{array}$ \\
\hline Cloke et al. [63] & UK & SPADI & Shoulder clinic & $\begin{array}{l}\text { Subacromial impingement } \\
\text { Mean age } 55,44 \% \text { male }\end{array}$ \\
\hline Croft et al. [54] & UK & SDQ-UK & GP & $\begin{array}{l}\text { Shoulder pain } \\
\text { Community mean age } 65,28 \% \text { male; General } \\
\text { practice attendees mean age } 51,48 \% \text { male }\end{array}$ \\
\hline Fan et al. [64] & USA & QuickDASH & Working population & $\begin{array}{l}\text { Shoulder pain } \\
\text { Mean age } 40,52 \% \text { male }^{a}\end{array}$ \\
\hline Godfrey et al. [53] & USA & SST & Hospital & $\begin{array}{l}\text { Rotator cuff disease } \\
\text { Mean age } 42,67 \% \text { male }\end{array}$ \\
\hline Hill et al. [34] & Australia & SPADI & General population & $\begin{array}{l}\text { Shoulder pain or stiffness } \\
\text { Mean age } 56,41 \% \text { male }\end{array}$ \\
\hline L'Insalata et al. [47] & USA & SRQ & Hospital & $\begin{array}{l}\text { Mixed types of shoulder pain } \\
\text { Mean age } 40,73 \% \text { male }\end{array}$ \\
\hline MacDermid et al. [39] & Canada & SPADI & General population & $\begin{array}{l}\text { Shoulder pain } \\
\text { Mean age } 44,49 \% \text { male }\end{array}$ \\
\hline Mintken et al. [52] & USA & QuickDASH & Physiotherapy & $\begin{array}{l}\text { Shoulder pain } \\
\text { Stable patients mean age } 44,59 \% \text { male; } \\
\text { Improved patients mean age } 39,66 \% \text { male }\end{array}$ \\
\hline Paul et al. [31] & UK & $\begin{array}{l}\text { SDQ } \\
\text { SDQ-UK } \\
\text { SPADI } \\
\text { SRQ }\end{array}$ & Shoulder clinic & $\begin{array}{l}\text { Shoulder pain } \\
\text { Mean age } 54,50 \% \text { male }\end{array}$ \\
\hline Roach et al. [6] & USA & SPADI & GP & $\begin{array}{l}\text { Shoulder pain } \\
\text { Mean age } 58,100 \% \text { male }\end{array}$ \\
\hline Staples et al. [40] & Australia & $\begin{array}{l}\text { SPADI } \\
\text { DASH }\end{array}$ & Physiotherapy & $\begin{array}{l}\text { Adhesive capsulitis } \\
\text { Mean age } 56,25 \% \text { male }\end{array}$ \\
\hline Tashjian et al. [51] & USA & SST & GP & $\begin{array}{l}\text { Rotator cuff disease } \\
\text { Mean age } 51,48 \% \text { male }\end{array}$ \\
\hline \multicolumn{5}{|l|}{ Dutch } \\
\hline van der Heiden et al. [5] & Netherlands & SDQ & Rehabilitation clinic & $\begin{array}{l}\text { Shoulder pain and stiffness } \\
\text { Mean age } 51,49 \% \text { male }\end{array}$ \\
\hline van Kampen et al. [50] & Netherlands & SST & Hospital & $\begin{array}{l}\text { Shoulder pain } \\
\text { Mean age } 39,72 \% \text { male }\end{array}$ \\
\hline Vermeulen et al. [48] & Netherlands & SRQ & Hospital & $\begin{array}{l}\text { Mixed types of shoulder pain } \\
\text { Mean age } 52,23 \% \text { male }\end{array}$ \\
\hline \multicolumn{5}{|l|}{ Norwegian } \\
\hline Ekeberg et al. [37] & Norway & SPADI & GP & $\begin{array}{l}\text { Rotator cuff disease } \\
\text { Mean age } 51,34 \% \text { male }\end{array}$ \\
\hline Ekeberg et al. [33] & Norway & SPADI & GP & $\begin{array}{l}\text { Rotator cuff disease } \\
\text { Mean age } 51,37 \% \text { male }\end{array}$ \\
\hline Haldorsen et al. [45] & Norway & DASH & Outpatient clinic & $\begin{array}{l}\text { Shoulder impingement } \\
\text { Mean age } 53,52 \% \text { male }\end{array}$ \\
\hline Tveita et al. [36] & Norway & SPADI & Hospital & $\begin{array}{l}\text { Adhesive capsulitis } \\
\text { Not reported }\end{array}$ \\
\hline Tveita et al. [35] & Norway & SPADI & Hospital & $\begin{array}{l}\text { Adhesive capsulitis } \\
\text { Mean age } 52,42 \% \text { male }\end{array}$ \\
\hline
\end{tabular}


Table 2 continued

\begin{tabular}{|c|c|c|c|c|}
\hline Study & Country & PROMs & Setting & Population \\
\hline \multicolumn{5}{|l|}{ Turkish } \\
\hline Bicer et al. [38] & Turkey & SPADI & Rehabilitation clinic & $\begin{array}{l}\text { Shoulder pain } \\
\text { Mean age 53,0\% male }\end{array}$ \\
\hline Dogu et al. [30] & Turkey & $\begin{array}{l}\text { SDQ } \\
\text { SPADI }\end{array}$ & Physiotherapy & $\begin{array}{l}\text { Shoulder impingement } \\
\text { Mean age 56, } 33 \% \text { male }\end{array}$ \\
\hline Ozsahin et al. [42] & Turkey & SDQ & Shoulder clinic & $\begin{array}{l}\text { Shoulder pain } \\
\text { Mean age 51, } 25 \% \text { male }\end{array}$ \\
\hline \multicolumn{5}{|l|}{ German } \\
\hline Offenbacher et al. [65] & Germany & DASH & Hospital & $\begin{array}{l}\text { Shoulder pain } \\
\text { Mean age 59, } 27 \% \text { male }\end{array}$ \\
\hline \multicolumn{5}{|l|}{ Danish } \\
\hline Christiansen et al. [32] & Denmark & SPADI & Hospital & $\begin{array}{l}\text { Shoulder pain } \\
\text { Mean age } 48,46 \% \text { male }\end{array}$ \\
\hline \multicolumn{5}{|l|}{ Spanish } \\
\hline Alvarez-Nemegyei et al. [66] & Mexico & SDQ & Hospital & $\begin{array}{l}\text { Subacromial impingement } \\
\text { Mean age } 55,20 \% \text { male }\end{array}$ \\
\hline \multicolumn{5}{|l|}{ Slovene } \\
\hline Jamnik et al. [41] & Slovenia & SPADI & Rehabilitation clinic & $\begin{array}{l}\text { Chronic shoulder complaints } \\
\text { Mean age } 56,29 \% \text { male }\end{array}$ \\
\hline \multicolumn{5}{|l|}{ Tamil } \\
\hline Jeldi et al. [67] & India & SPADI & Physiotherapy & $\begin{array}{l}\text { Shoulder pain or dysfunction } \\
\text { Mean age } 49,48 \% \text { male }\end{array}$ \\
\hline
\end{tabular}

${ }^{a}$ Based on whole cohort, not separately reported for the section of interest

related constructs were higher than with unrelated constructs.

Construct cross-cultural validity Only studies that were rated as being of poor methodology have been performed.

\section{Responsiveness}

There is moderate positive evidence for responsiveness of the English version (AUC ranging between 0.74 and 0.87 ) $[31,40]$ and the Norwegian version $(\mathrm{AUC}=0.84$ or 0.92 depending on the follow-up period) [33].

\section{Shoulder Disability Questionnaire (SDQ)}

The SDQ is 16-item pain-related disability questionnaire that was originally developed in Dutch. Response options are "yes," "no" or "not applicable," resulting in a total score which ranges from 0 to 100 , with a higher score indicating more severe disability [4]. It takes about 2 [30, $31]$ to $4 \mathrm{~min}$ to complete, and patients indicated the SDQ as (very) easy to complete [5, 30, 31]. One study assessed whether there were signs of floor or ceiling effects; however, they did not report the data needed to give a proper indication of it [5].

\section{Reliability}

Internal consistency Only studies that were rated as being of poor methodology have been performed.

Reliability There were no sound methodological studies evaluating reliability, except for the Turkish version, which showed limited positive evidence, with a Pearson correlation coefficient of 0.88 for the total score [42].

Measurement error There were no studies evaluating the measurement error.

\section{Validity}

Content validity The evidence regarding content validity of the original SDQ is indeterminate, as the questions are not aimed at the originally described construct (see Table 4).

Construct structural validity There were no studies evaluating structural validity.

Construct hypothesis testing There is limited positive evidence for the Dutch version [43] and limited negative evidence 
Table 3 Overview of PROMs used with their originally described construct and an example of questions used

\begin{tabular}{|c|c|c|}
\hline PROM & $\begin{array}{l}\text { Description of the construct by the original author (and the } \\
\text { author of a study assessing content validity) }\end{array}$ & Example of used questions \\
\hline SPADI & Pain and disability [6] & $\begin{array}{l}\text { 1. How severe is your pain when.... When lying on the } \\
\text { involved side? } \\
\text { 2. How much difficulty did you have.... washing your back? }\end{array}$ \\
\hline SDQ & $\begin{array}{l}\text { Functional status limitation [5] } \\
\text { Pain-related disability [43] }\end{array}$ & $\begin{array}{l}\text { 1. My shoulder hurts when I lie on it: } \mathrm{Y} / \mathrm{N} \\
\text { 2. My shoulder is painful when I open or close a door: } \mathrm{Y} / \mathrm{N}\end{array}$ \\
\hline DASH & $\begin{array}{l}\text { Symptoms and functional status focused on physical function. } \\
\text { The items tap upper extremity-related symptoms and measure } \\
\text { functional status at the level of disability. Disability is defined } \\
\text { as "difficulty doing activities in any domain of life (the } \\
\text { domains typical for one's age-sex group) due to a health or } \\
\text { physical problem" [7] }\end{array}$ & $\begin{array}{l}\text { Please circle the number that best describes your physical } \\
\text { ability in the past week. Did you have any difficulty: } \\
\text { 1. Using your usual technique for your work? } \\
\text { 2. Doing your usual work because of arm, shoulder or hand } \\
\text { pain? } \\
\text { No difficulty (1)-Unable (5) }\end{array}$ \\
\hline SRQ & Symptoms and function [47] & $\begin{array}{l}\text { The following questions refer to pain: } \\
\text { 1. During the past month, how would you describe the usual } \\
\text { pain in your shoulder during activities? Very severe (1)- } \\
\text { None (5) } \\
\text { The following questions refer to daily activities: } \\
\text { 1. During the past month, how much difficulty have you had in } \\
\text { each of the following activities due to your shoulder; putting } \\
\text { on or removing a pullover sweater or shirt? Unable (1)—No } \\
\text { difficulty (5) }\end{array}$ \\
\hline SST & Functional limitations of the affected shoulder [49] & $\begin{array}{l}\text { 1. Can you reach the small of your back to tuck in your shirt } \\
\text { with your hand? Y/N } \\
\text { 2. Can you place your hand behind your head with the elbow } \\
\text { straight out to the side? Y/N }\end{array}$ \\
\hline QuickDASH & $\begin{array}{l}\text { Physical function and symptoms in persons with any or } \\
\text { multiple musculoskeletal disorders of the upper limb [58] }\end{array}$ & $\begin{array}{l}\text { Please rate your ability to do the following activities in the last } \\
\text { week by circling the number below the appropriate response } \\
\text { 1. Open a tight or new jar } \\
\text { 2. Do heavy household chores (e.g., wash walls, floors) } \\
\text { No difficulty (1)_Unable (5) }\end{array}$ \\
\hline SDQ-UK & Disability associated with shoulder symptoms [54] & $\begin{array}{l}\text { 1. Because of my shoulder, I move my arm or hand with some } \\
\text { difficulty: Y/N } \\
\text { 2. I do not bath myself completely because of my shoulder: } \\
\text { Y/N }\end{array}$ \\
\hline
\end{tabular}

for the English version (as three out of the seven expected positive correlations measured were below 0.50) [31].

Construct cross-cultural validity No studies specifically assessed cross-cultural validity.

\section{Responsiveness}

There is moderate positive evidence for the Dutch version $(\mathrm{AUC}=0.84)$ [4] and limited positive evidence for the English version (AUC $=0.77)$ [31].

\section{Disability of arm, shoulder and hand (DASH)}

The DASH is designed to measure symptoms and physical functioning in patients with pain in the arm, shoulder or hand. It consists of 30 items, and the response options for each item are presented as 5-point Likert scales. The total score ranges from 0 to 100 [7]. We did not find studies reporting any item on feasibility. No floor or ceiling effects were detected [44, 45].

\section{Reliability}

Internal consistency Only studies that were rated as being of poor methodology have been performed.

Reliability There is limited positive evidence for the Norwegian version (ICC $=0.89$ ) [45].

Measurement error The result of the only study with fair methodology evaluating measurement error is indeterminate, as they did not provide the MIC; the SDC, however, was 6.7 points for the Norwegian version [45]. 
Table 4 Methodological quality of each study per measurement property

\begin{tabular}{|c|c|c|c|c|c|c|c|c|}
\hline Study & $\begin{array}{l}\text { Internal } \\
\text { consistency }\end{array}$ & Reliability & $\begin{array}{l}\text { Measurement } \\
\text { error }\end{array}$ & $\begin{array}{l}\text { Content } \\
\text { validity }\end{array}$ & $\begin{array}{l}\text { Structural } \\
\text { validity }\end{array}$ & $\begin{array}{l}\text { Hypotheses } \\
\text { testing }\end{array}$ & $\begin{array}{l}\text { Cross-cultural } \\
\text { validity/*only a } \\
\text { translation }\end{array}$ & Responsiveness \\
\hline \multicolumn{9}{|c|}{ SPADI developed in English } \\
\hline $\begin{array}{l}\text { Bicer et al. } \\
\text { [38] }\end{array}$ & Poor & Fair & & & & Fair & & \\
\hline $\begin{array}{c}\text { Christiansen } \\
\text { et al. [32] }\end{array}$ & Poor & Poor & Poor & & & Fair & Poor & \\
\hline $\begin{array}{l}\text { Cloke et al. } \\
\text { [63] }\end{array}$ & & Poor & & & & Poor & & Poor \\
\hline $\begin{array}{l}\text { Dogu et al. } \\
{[30]}\end{array}$ & & & & & & & & Poor \\
\hline $\begin{array}{l}\text { Ekeberg et al. } \\
\text { [37] }\end{array}$ & Poor & Good & Good & & & Fair & & \\
\hline $\begin{array}{l}\text { Ekeberg et al. } \\
\text { [33] }\end{array}$ & & & & & & & & Good \\
\hline Hill et al. [34] & Excellent & & & & Good & Poor & & \\
\hline $\begin{array}{l}\text { Jamnik et al. } \\
\text { [41] }\end{array}$ & Poor & Poor & & & Poor & Fair & Fair* & Poor \\
\hline Jeldi et al. [67] & Poor & Poor & & & & Poor & Poor & \\
\hline $\begin{array}{r}\text { MacDermid } \\
\text { et al. [39] }\end{array}$ & Fair & & & & Fair & Fair & & Poor \\
\hline Paul et al. [31] & & & & & & Fair & & Fair \\
\hline Roach et al. [6] & Poor & Poor & & & Poor & Poor & & Poor \\
\hline $\begin{array}{l}\text { Staples et al. } \\
\text { [40] }\end{array}$ & & & & & & Fair & & Fair \\
\hline $\begin{array}{l}\text { Tveita et al. } \\
\text { [36] }\end{array}$ & & Fair & Fair & & & & Fair* & Poor \\
\hline $\begin{array}{l}\text { Tveita et al. } \\
\text { [35] }\end{array}$ & Fair & & & & Fair & & & \\
\hline \multicolumn{9}{|c|}{ SDQ developed in Dutch } \\
\hline $\begin{array}{l}\text { Alvarez- } \\
\text { Nemegyei } \\
\text { et al. [66] }\end{array}$ & Poor & Poor & & & & & Poor & \\
\hline $\begin{array}{l}\text { Dogu et al. } \\
\text { [30] }\end{array}$ & & & & & & & & Poor \\
\hline $\begin{array}{l}\text { van der Heiden } \\
\text { et al. [5] }\end{array}$ & & & & & & & & Fair \\
\hline $\begin{array}{l}\text { Ozsahin et al. } \\
\text { [42] }\end{array}$ & Poor & Fair & & & & Poor & Poor* & \\
\hline Paul et al. [31] & & & & & & Fair & & Fair \\
\hline $\begin{array}{l}\text { van der Windt } \\
\text { et al. [4] }\end{array}$ & & & & & & & & Good \\
\hline $\begin{array}{l}\text { de Winter et al. } \\
\text { [43] }\end{array}$ & Poor & & & Excellent & & Fair & & \\
\hline \multicolumn{9}{|c|}{ DASH developed in English } \\
\hline $\begin{array}{l}\text { Beaton et al. } \\
\text { [44] }\end{array}$ & & & & & & Fair & & \\
\hline $\begin{array}{l}\text { Haldorsen } \\
\text { et al. [45] }\end{array}$ & Poor & Fair & Fair & & & Fair & & \\
\hline $\begin{array}{l}\text { Offenbacher } \\
\text { et al. [65] }\end{array}$ & Poor & Poor & & & & Fair & Poor* & \\
\hline $\begin{array}{l}\text { Staples et al. } \\
\text { [40] }\end{array}$ & & & & & & Fair & & Fair \\
\hline
\end{tabular}


Table 4 continued

\begin{tabular}{|c|c|c|c|c|c|c|c|c|}
\hline Study & $\begin{array}{l}\text { Internal } \\
\text { consistency }\end{array}$ & Reliability & $\begin{array}{l}\text { Measurement } \\
\text { error }\end{array}$ & $\begin{array}{l}\text { Content } \\
\text { validity }\end{array}$ & $\begin{array}{l}\text { Structural } \\
\text { validity }\end{array}$ & $\begin{array}{l}\text { Hypotheses } \\
\text { testing }\end{array}$ & $\begin{array}{l}\text { Cross-cultural } \\
\text { validity/*only a } \\
\text { translation }\end{array}$ & Responsiveness \\
\hline \multicolumn{9}{|c|}{ SRQ developed in English } \\
\hline $\begin{array}{l}\text { L'Insalata } \\
\text { et al. [47] }\end{array}$ & Poor & Poor & & & & Poor & & \\
\hline Paul et al. [31] & & & & & & Fair & & Fair \\
\hline $\begin{array}{l}\text { Vermeulen } \\
\text { et al. [48] }\end{array}$ & Poor & Fair & & & & Poor & Excellent* & \\
\hline \multicolumn{9}{|c|}{ SST developed in English } \\
\hline $\begin{array}{l}\text { Godfrey et al. } \\
\text { [53] }\end{array}$ & & & & & & Poor & & \\
\hline $\begin{array}{c}\text { van Kampen } \\
\text { et al. [50] }\end{array}$ & Excellent & Fair & Fair & & Excellent & Good & Fair* & \\
\hline $\begin{array}{l}\text { Tasjian et al. } \\
\text { [51] }\end{array}$ & & & & & & & & Poor \\
\hline \multicolumn{9}{|c|}{ QuickDASH developed in English } \\
\hline Fan et al. [64] & & & & & & Poor & & \\
\hline $\begin{array}{l}\text { Mintken et al. } \\
\text { [52] }\end{array}$ & & Poor & Poor & & & & & Fair \\
\hline \multicolumn{9}{|c|}{ SDQ-UK developed in English } \\
\hline Croft et al. [54] & & & & Poor & & Poor & & \\
\hline Paul et al. [31] & & & & & & Fair & & Fair \\
\hline
\end{tabular}

* only a translation

\section{Validity}

Content validity There were no studies evaluating content validity.

Construct structural validity There were no studies evaluating structural validity.

Construct hypothesis testing There is moderate positive evidence for construct hypothesis testing of the English version [40, 44] and limited positive evidence for the German [46] and Norwegian version [45].

Construct cross-cultural validity No studies specifically assessed cross-cultural validity.

\section{Responsiveness}

There is limited positive evidence for the English version for responsiveness $(\mathrm{AUC}=0.71-0.86$ depending on the anchor used) [40].

\section{Shoulder Rating Questionnaire (SRQ)}

The SRQ was developed to measure the severity of symptoms related to and the functional status of the shoulder. It covers seven domains including 21 items-the total score ranges between 17 and 100 [47] — takes about 4 [31] to 7 [48] minutes to complete and is moderately easy to complete according to patients [31]

\section{Reliability}

Internal consistency Only studies that were rated as being of poor methodology have been performed.

Reliability There was limited positive evidence for the reliability of the Dutch version $(\mathrm{ICC}=0.85)$ [48].

Measurement error There were no studies evaluating the measurement error.

\section{Validity}

Content validity There were no studies evaluating content validity.

Construct structural validity There were no studies evaluating structural validity.

Construct hypothesis testing There was limited positive evidence for the English SRQ [31]. 
Table 5 Comparator instrument in case of hypothesis testing

Study Comparator instruments and correlations

SPADI

Bicer et al. [38]

Christiansen et al.

Ekeberg et al. [37]

Jamnik et al. [41]

MacDermid et al.

Paul et al. [31]

Staples et al. [40]

SDQ

Paul et al. [31]

de Winter et al.

\section{DASH}

Beaton et al. [44]

Haldorsen et al. [45]

Offenbacher et al. [65]

Staples et al. [40]

SRQ

Paul et al. [31]

SST

Kampen van et al. [50]

SDQ-UK

Paul et al. [31]
Convergent: the Spearman correlation with the HAQ total score was 0.67 and 0.65 with VAS during AROM

Known groups: those currently working, despite their shoulder pain, were found to have significantly lower scores than those not working; the mean difference was -18.3 (95\% CI -29.4 to -7.2 )

Convergent: the Spearman correlation with the OSS total score was $0.57,-0.67$ for the WORC total, -0.75 with WORC physical, -0.46 with WORC Sports, -0.55 with WORC Work and -0.69 with WORC Lifestyle

Divergent: the Spearman correlation between the SPADI and the WORC emotions was -0.31

Known groups: participants who differed in the severity of the perceived disability self-rating (mild-moderate-severe) differed significantly in the SPADI score in the presumed order

Known groups: patients who had diagnosed shoulder problems and those on pain mediation reported significantly higher pain and disability scores. Convergent: convergent scales (Home management 0.59, Work -0.10 , Physical dimension 0.51) of the SIP showed a moderate correlation, except the work scale

Divergent: divergent (emotional) scales of the SIP showed low correlations $(0.17-0.33)^{\mathrm{a}}$

Convergent: the spearman correlation with other shoulder PROMs was: 0.57 for the SDQ-UK, 0.33 with the SDQ and 0.83 with the SRQ. The correlation with Difficulty VAS $0.62^{\mathrm{a}}$

Convergent: the Pearson correlation with other shoulder PROMs was: 0.55 with the DASH and 0.65 with the Croft index. Correlations with generic PROMs were: 0.17 with PET, 0.60 with Pain and 0.55 with the HAQ

Convergent: the Spearman correlation with other shoulder PROMs was: 0.55 for the SDQ-UK, 0.33 with the SPADI and 0.43 with the SRQ. The correlation with Difficulty VAS $0.47^{\mathrm{a}}$

Known groups: significant differences in the SDQ scores $(p<0.001)$ were found for subgroups with different pain severity, ability to perform activities in daily life, mobility, muscle force, and levels of disability according to the physical therapists. Convergent: the Spearman correlation with severity of disability was 0.58 , and degree of difficulty for the main functional limitation was $0.32^{\mathrm{a}}$

Known groups: those currently working with their upper limb condition and able to continue doing so had significantly lower disability than those who were not able to work $(26.8$ vs. $50.7, t=-7.51, p<0.001)$. Statistically significant differences were also found between those who were able to do all they want to do as opposed to those who were not able to do so (23.6 vs. 47.1, $t=-5.81, p<0.0001)$. Convergent: The Spearman correlation with the overall rating of the problem was 0.68 , with the ability to function 0.85 , with the ability to work 0.76 , with Brigham symptoms 0.71 and 0.90 with Brigham symptoms. The Spearman correlation with another shoulder PROM 0.76 with the SPADI pain scale and 0.83 with the SPADI function scale ${ }^{\mathrm{a}}$

Convergent: the Pearson correlation with the SPADI was 0.75 and with the NPRS 0.58 . The correlations with components of the SF-36 were: physical functioning -0.48 , bodily pain -0.62 , and physical component summary $-0.59$

Divergent: the Pearson correlation with the mental component summary score of the SF- 36 was -0.17 and -0.35 with the social functioning scale of the SF-36

Convergent: the Spearman correlation with the HAQ was 0.81 , with the SF-36 physical functioning component -0.58 , and with global impact $0.76^{\mathrm{a}}$

Convergent: the Pearson correlation with other shoulder PROMs was: 0.55 with the SPADI and 0.65 with the Croft index. Correlations with generic PROMs were: 0.20 with PET and 0.54 with the HAQ ${ }^{\mathrm{a}}$

Convergent: the spearman correlation with other shoulder PROMs was: 0.72 for the SDQ-UK, 0.83 with the SPADI and 0.43 with the SDQ. The correlation with Difficulty VAS $0.60^{\mathrm{a}}$

Convergent: the Pearson correlation with other shoulder PROMs was: 0.74 with the OSS, 0.59 with the CM and 0.74 with the DASH. The correlation with the SF-36 subscale physical functioning was 0.56

Convergent: the Spearman correlation with other shoulder PROMs was: 0.72 for the SRQ, 0.57 with the SPADI and 0.55 with the SDQ. The correlation with Difficulty VAS $0.41^{\mathrm{a}}$

${ }^{a} \mathrm{ROM}$, pain alone and the EQ5D were considered to be inappropriate comparators and were therefore excluded in the rating process 
Table 6 Best evidence synthesis

\begin{tabular}{|c|c|c|c|c|c|c|c|c|}
\hline PROM & $\begin{array}{l}\text { Internal } \\
\text { consistency }\end{array}$ & Reliability & $\begin{array}{l}\text { Measurement } \\
\text { error }\end{array}$ & $\begin{array}{l}\text { Content } \\
\text { validity }\end{array}$ & $\begin{array}{l}\text { Structural } \\
\text { validity }\end{array}$ & $\begin{array}{l}\text { Hypotheses } \\
\text { testing }\end{array}$ & $\begin{array}{l}\text { Cross-cultural } \\
\text { validity }\end{array}$ & Responsiveness \\
\hline \multicolumn{9}{|l|}{ English } \\
\hline SPADI & +++ & $?$ & 0 & 0 & ++ & ++ & 0 & ++ \\
\hline DASH & 0 & 0 & 0 & 0 & 0 & ++ & 0 & + \\
\hline SDQ-UK & 0 & 0 & 0 & $?$ & 0 & + & 0 & + \\
\hline SRQ & $?$ & $?$ & 0 & 0 & 0 & + & 0 & + \\
\hline SDQ-English & 0 & 0 & 0 & 0 & 0 & - & 0 & + \\
\hline SST & 0 & 0 & 0 & 0 & 0 & $?$ & 0 & $?^{\mathrm{d}}$ \\
\hline QuickDASH & 0 & 0 & 0 & 0 & 0 & $?$ & 0 & 0 \\
\hline \multicolumn{9}{|l|}{ Dutch } \\
\hline SST-Dutch & +++ & + & $?^{\mathrm{a}}$ & 0 & +++ & ++ & 0 & 0 \\
\hline SDQ & $?$ & 0 & 0 & $?^{\mathrm{b}}$ & 0 & + & 0 & ++ \\
\hline $\begin{array}{l}\text { Quick DASH- } \\
\text { Dutch }\end{array}$ & 0 & $?$ & $?$ & 0 & 0 & 0 & 0 & + \\
\hline SRQ-Dutch & $?$ & + & 0 & 0 & 0 & $?$ & 0 & 0 \\
\hline \multicolumn{9}{|l|}{ Norwegian } \\
\hline $\begin{array}{l}\text { SPADI- } \\
\text { Norwegian }\end{array}$ & + & ++ & $?^{\mathrm{a}}$ & 0 & - & + & 0 & ++ \\
\hline $\begin{array}{l}\text { DASH- } \\
\text { Norwegian }\end{array}$ & $?$ & + & $?^{\mathrm{a}}$ & 0 & 0 & + & 0 & 0 \\
\hline \multicolumn{9}{|l|}{ Turkish } \\
\hline SPADI-Turkish & $?$ & + & 0 & 0 & 0 & + & 0 & $?$ \\
\hline SDQ-Turkish & $?$ & + & 0 & 0 & 0 & $?$ & 0 & $?$ \\
\hline \multicolumn{9}{|l|}{ German } \\
\hline DASH-German & $?$ & $?$ & 0 & 0 & 0 & + & 0 & 0 \\
\hline \multicolumn{9}{|l|}{ Danish } \\
\hline SPADI-Danish & $?$ & $?$ & $?$ & 0 & 0 & $?^{\mathrm{c}}$ & $?$ & 0 \\
\hline \multicolumn{9}{|l|}{ Spanish } \\
\hline $\begin{array}{l}\text { SDQ-Spanish } \\
\text { (Mexican) }\end{array}$ & $?$ & $?$ & 0 & 0 & 0 & 0 & $?$ & 0 \\
\hline \multicolumn{9}{|l|}{ Slovene } \\
\hline SPADI-Slovene & $?$ & $?$ & 0 & 0 & $?$ & $?^{\mathrm{c}}$ & 0 & $?$ \\
\hline \multicolumn{9}{|l|}{ Tamil } \\
\hline SPADI-Tamil & ? & ? & 0 & 0 & 0 & ? & ? & 0 \\
\hline
\end{tabular}

${ }^{a}$ Despite fair/good methodology, the level of evidence could not be determined as the appropriate measurement properties were not provided

${ }^{\mathrm{b}}$ Despite fair/good methodology, the level of evidence could not be determined as the originally described construct differed from the construct described in the current study

${ }^{c}$ Despite fair/good methodology, the level of evidence could not be determined as unclear, as they confirmed their hypothesis with known group validity, but did not assess whether the correlations with related constructs were higher than with unrelated constructs

d This study only evaluated the minimal clinical difference

Construct cross-cultural validity No studies specifically assessed cross-cultural validity.

\section{Responsiveness}

There was limited positive evidence for the responsiveness of the English SRQ (AUC = 0.85) [31].

\section{Simple shoulder test (SST)}

The SST was developed to measure functional limitations in patients with shoulder dysfunction. It consists of 12 items, and the response options are dichotomous. The total score ranges between 0 and 12 [49]. We did not find studies reporting any item on feasibility. 
No floor or ceiling effects were detected [50].

\section{Reliability}

Internal consistency There was strong positive evidence for the Dutch SST with a Cronbach Alpha of 0.78 [50].

Reliability There was limited positive evidence for the reliability of the Dutch SST (ICC $=0.92$ ) [50].

Measurement error The result of the only study with fair methodology evaluating measurement error is indeterminate, as they did not provide the MIC; the SDC, however, was 3.3 [50].

\section{Validity}

Content validity There were no studies evaluating content validity.

Construct structural validity There was strong evidence for the unidimensionality of the Dutch SST. Confirmatory factor analysis of a 1-factor model showed a moderate fit (CFI 0.94, TLI 0.93, RMSEA 0.07), and three items showed relatively low factor loadings [50].

Construct hypothesis testing There is moderate positive evidence for construct hypothesis testing of the Dutch SST [50].

Construct cross-cultural validity No studies specifically assessed cross-cultural validity.

\section{Responsiveness}

There were no studies judged as having a sound methodology evaluating the English version. One study on the English SST only calculated the minimal clinically important difference, but did not assess the responsiveness [51].

\section{QuickDASH}

The QuickDASH is an 11-item questionnaire that addresses symptoms and physical function in people with disorders of the arm, shoulder or hand. It provides a summative percentage score, with 100 indicating the most disability [52]. We did not find studies reporting on feasibility. No floor or ceiling effects were detected [53].

\section{Reliability}

Internal consistency There were no studies evaluating internal consistency.
Reliability Only studies that were rated as being of poor methodology have been performed.

Measurement error Only studies that were rated as being of poor methodology have been performed.

\section{Validity}

Content validity There were no studies evaluating content validity.

Construct structural validity There were no studies evaluating structural validity.

Construct hypothesis testing Only studies that were rated as being of poor methodology have been performed.

Construct cross-cultural validity No studies specifically assessed cross-cultural validity.

\section{Responsiveness}

There was limited positive evidence for responsiveness in the Dutch version (AUC $=0.82)[52]$.

\section{Shoulder Disability Questionnaire (SDQ-UK)}

The SDQ-UK is a 22-item questionnaire [54]. The questionnaire contains some statements that people have used to describe themselves when they have trouble with their shoulder. Participants are asked to answer "yes" or "no" depending on whether they recognize the statement as applying to them, with a total score ranging between 0 and 100. It takes about 3 min to complete and patients describe it as easy to understand [31].

\section{Reliability}

Internal consistency There were no studies evaluating internal consistency.

Reliability There were no studies evaluating reliability.

Measurement error There were no studies evaluating the measurement error.

\section{Validity}

Content validity Only studies that were rated as being of poor methodology have been performed.

Construct structural validity There were no studies evaluating structural validity. 
Construct hypothesis testing There was limited positive evidence for construct hypothesis testing [31].

Construct cross-cultural validity No studies specifically assessed cross-cultural validity.

\section{Responsiveness}

There was limited positive evidence for the responsiveness $(\mathrm{AUC}=0.77)[31]$.

\section{Recommended PROMS per language}

\section{English}

All seven PROMs were available and assessed in English. For English users, we recommend using the English SPADI as it was rated best in the best evidence synthesis. It consists of two factors: There is strong positive evidence for the internal consistency and moderate evidence for construct hypothesis testing and the responsiveness.

\section{Dutch}

Four questionnaires were available and assessed in Dutch in this specific population. The SDQ was developed in Dutch, and the other three were developed in English. Both the SDQ and SST showed acceptable ratings in the best evidence synthesis. There was strong evidence for the reliability as well as for the construct validity for the Dutch SST. Strong positive evidence was found for the internal consistency and limited positive evidence for the reliability of the Dutch SST, and inconclusive evidence for the measurement error. The construct validity of the SST was strong, as there was strong evidence for the unidimensionality and moderate positive evidence for construct hypothesis testing.

There is limited positive evidence for construct hypothesis testing of the Dutch SDQ, and there is moderate positive evidence for responsiveness. We recommend choosing between either the SST or the SDQ depending on the purpose of its use.

\section{Norwegian}

Out of the two available instruments, the SPADI showed the best ratings. There is moderate positive evidence for the reliability and inconclusive evidence for the measurement error. There was limited evidence that the Norwegian SPADI did not follow the original factor structure and limited positive evidence for the internal consistency. There was limited positive evidence for construct hypothesis testing and moderate positive evidence for the responsiveness.

\section{Turkish}

In Turkish, both the SDQ and the SPADI were evaluated, and both only showed limited evidence; however, the SPADI also had limited evidence for construct hypothesis testing instead of only limited evidence for reliability. We therefore recommend using the SPADI, however, caution is advised.

\section{German}

We only found one study using a PROM in German when using our search criteria. There is limited positive evidence for the construct hypothesis of the German DASH. We recommend using the DASH in the German language; however, it is important to be aware of the lack of information available about this PROM in German.

\section{Other languages}

In Danish, Tamil and Slovene, the only instrument evaluated was the SPADI, in Spanish the only questionnaire assessed was the SDQ. For all four languages, we only found studies with poor methodology or information was missing regarding a measurement property. We could therefore not make a recommendation in these languages.

\section{Discussion}

The SPADI has been the most frequently evaluated questionnaire in this review on patients with shoulder pain and its measurement properties seem adequate apart from a lack of information regarding its reliability, measurement error and content validity. For English users, we recommend its use, as this is the PROM with the best measurement properties.

For Norwegian users, the SPADI is recommended, as well for Turkish users, although for the latter caution is advised as the evidence is limited and information on some measurement properties is lacking. Dutch users could use either the SDQ or the SST, depending on the intended purpose. Germans could use the DASH, although caution is advised, as there is still a lack of information regarding many measurement properties.

In Danish, Spanish, Tamil and Slovene, the evaluated PROMs were not yet of acceptable validity. We found no studies concerning PROMs in other languages, which met our inclusion criteria.

\section{Comparison with the literature}

One systematic review, assessing the methodological quality of measurement properties of shoulder PROMs, 
concluded that the DASH received the best ratings [8]. This is in contrast with our findings. A possible reason for this difference is the search period. Most studies reporting on the SPADI in our review were published after the search period (2002) of the previous review. Moreover, we excluded studies evaluating the DASH that did not report their results for shoulder pain patients separately.

Another recent review concluded that all of the included PROMs showed acceptable psychometric properties [10]. This study recommended PROMs that we excluded in our review [10]. The methodological quality of the studies included ranged from 33.3 to $95.9 \%$. No evidence synthesis was performed, and the psychometric properties per PROM were presented but without the methodological quality per study [10].

A review that evaluated the DASH, ASES, SPADI and SST only concluded that their measurement properties were acceptable and that none of the questionnaires was superior or could be recommended over the other. The quality of the individual studies ranged from 25 to $96 \%$ [9]. This study presented the psychometric properties of all included studies, but did not use the methodological quality of the studies themselves in their conclusions about the psychometric properties of an instrument.

Our search strategy was designed to be highly sensitive rather than specific, resulting in a higher number of hits (3421) compared to other reviews [8-10, 12]. Two reviews did not describe their search strategy [11, 13], and two reviews also included studies that were not designed to validate a PROM $[9,10]$.

Most importantly, these reviews used an unspecified study population (e.g., including postoperative patients), included PROMs focused on a specific pathology (e.g., instability) and PROMs that included a physical component. We specified our study population and excluded studies that did not report their results for patients with shoulder pain separately. As a consequence, we excluded a high amount of studies that were focused on the DASH. Due to our strict selection criteria, we also excluded a number of well-known PROMs, due to our specific research question, such as the WOSI, a PROM that is designed specifically for patients with instability, or the ASES, which includes a physical component.

The major flaws we found with respect to the methodology are comparable with another study on measurement properties of neck pain and disability questionnaires [55]. For internal consistency, most studies did not measure the unidimensionality of the scale. The time interval and the sample size were the main problems within the reliability category, and sample size or performing a confirmatory analysis for cross-cultural validity.

\section{Strengths and limitations}

We excluded two studies because we could not retrieve them as full text papers. One was written in Turkish. This could potentially have led to selection bias. However, the leading journals, and consequently the most important papers, are published in English.

We pooled our results by language rather than by country although we recognize that cultural differences may exist between countries. This means that for the English versions of PROMs, we pooled data from the UK, USA, Canada and Australia, hereby neglecting possible cultural differences. If countries are very close in location/culture/use of language and the text does not contain wording about education, health systems, brand names or IT, it is acceptable to use the same language version and to pool data from trials [56]. With respect to this, we assumed there are no insurmountable differences between the UK, USA, Canada and Australia. Moreover, our results did not show inconsistencies regarding measurement properties.

We excluded patients with generic and serious conditions (e.g., rheumatoid arthritis, fractures) and postoperative patients; therefore, our results cannot be extrapolated to these kinds of patients. The DASH is designed for patients with upper extremity disorders. Our conclusion on the DASH and its measurement properties are based on patients with shoulder pain only. Our results are therefore incomplete regarding the measurement properties of the DASH itself and cannot be extrapolated to other groups of patients on which the DASH can be used.

\section{Considerations regarding the results}

We found that content validity of most PROMs is still unknown (a PROM should have evidence supporting its content validity, including evidence that patients and/or experts consider the content of the PROM relevant and comprehensive for the concept, population, and aim of the measurement application [57]), although content validity is often considered to be the most important measurement property [57]. We could only rate the SDQ and the SDQUK on content validity, as some development studies did not involve patients or did not present their results separately for patients with shoulder pain $[6,7,47,49,58,59]$. Originally, the construct of the SDQ was described as "functional status" [5], but the items used were focussed on pain, e.g., "my shoulder hurts when I lie on it," resulting in a lack of face validity. However, the study which assessed the content validity of the SDQ, used "pain related disability" [43] as the construct to be measured, which would be a more appropriate term. It is therefore important to clearly describe the construct to be measured. 
All other PROMs did not show much discrepancy between the described construct and its items. However, in case of the SRQ, SDQ-UK and SST, the construct was not described in generally accepted terms (ICF terminology) or an extensive description, which makes it difficult to assess whether the items are an adequate reflection of the construct to be measured.

Most studies focused on validity. However, internal consistency, reliability and responsiveness were also well represented. For hypothesis testing, various comparator instruments were used: shoulder PROMs focused on activity limitation/pain-related disability (e.g., SDQ, SDQUK, SRQ, DASH, SPADI), known groups (e.g., medication, specific diagnosis, currently working), general PROMs (e.g., pain intensity, HAQ) and range of motion. An important aspect of the methodological quality assessment is whether the comparator instruments measure the same construct and show adequate measurement properties. We considered that range of motion measures a different construct and we therefore rated studies that solely used range of motion as a comparator instrument as being of poor methodology. We also excluded the comparisons with pain alone and the EQ5D as these also measure a different construct, although in most cases this did not influenced the final ratings.

\section{Recommendations for future research}

Further research is recommended to fill the gaps in knowledge regarding the measurement properties of shoulder-specific PROMs, especially with respect to their content validity, starting with a clear description of the construct, but also whether all items seem to be relevant to patients.

Although all of the evaluated instruments were developed in the 1990s, none of these PROMs showed strong positive evidence for all measurement properties after 20 years of research. Meanwhile, knowledge regarding the development of a PROM has increased and instrument developers must articulate how a particular conceptual framework guided their construct selection, item development (including in-depth interviews and focus groups with patients and experts in the field) and psychometric testing [60]. Also, important issues concerning the limitation of functional activities have changed over time, e.g., computer use is nowadays completely integrated into everyday life, but this is not included in most PROMs. Not only relevant items have been changed, but also the available methodology and technology have reached a new level of sophistication, including "modern" psychometric techniques of item banking, item response theory (IRT) and computer-adaptive testing (CAT) [60]. Recently, the Patient-Reported Outcomes Measurement Information
System (PROMIS) was developed using sample qualitative input from patients and IRT methods, to construct and evaluate a preliminary item bank for measuring physical function [61]. At this moment, there are upper-extremity and mobility subdomain scores from the PROMIS physical functioning adult item bank [62].

Computer-adaptive testing has tremendous potential for yielding precise PROM assessment quickly and with significantly reduced respondent burden [60]. The methods of the PROMIS project are likely to substantially improve measures of physical function and to increase the efficiency of their administration using CAT [61].

We therefore propose to develop a new shoulder PROM focused on activity limitations, or evaluate the usefulness of an instrument such as the upper extremity PROMIS scale on patients with shoulder pain, taking new knowledge and techniques into account.

Our study showed that there is a lack of high-quality studies measuring cross-cultural validation. Most often PROMs are being translated, and some measurement properties are assessed. We feel it is of great importance to perform cross-cultural validation for PROMs [57].

\section{Compliance with ethical standards}

Conflict of interest None.

Open Access This article is distributed under the terms of the Creative Commons Attribution 4.0 International License (http://crea tivecommons.org/licenses/by/4.0/), which permits unrestricted use, distribution, and reproduction in any medium, provided you give appropriate credit to the original author(s) and the source, provide a link to the Creative Commons license, and indicate if changes were made.

\section{Appendix}

("Shoulder Pain"/OR ((pain* OR complaint* OR disorder* OR lesion* OR injur* OR stiff* OR tight* OR patholog* OR impingem* OR disease*) ADJ3 shoulder*).ab,ti.) OR ((shoulder/OR shoulder joint/OR (shoulder* OR (joint* ADJ3 (glenohumeral OR humeroscapular OR scapulohumeral OR "scapulo humeral"))).ab,ti.) AND (pain/OR "Wounds and Injuries"/OR "Arm Injuries"/OR ((functional ADJ3 (disorder* OR illness* OR impairment* OR limitation* OR disabilit* OR status* OR complaint*)) OR ((activit* OR participat*) ADJ6 (limit* OR complicat* OR interfer*)) OR (Disabilit* ADJ3 Evaluat*)).ab,ti.)) AND (exp questionnaires/OR (questionnaire* OR ((self OR patient*) ADJ3 report*) OR PRO OR PROM).ab,ti.) AND (instrumentation.xs. OR methods.xs. OR validation studies.pt. OR comparative study.pt. OR exp psychometrics/OR exp "outcome assessment (health care)"/OR observer variation/OR exp Health Status Indicators/OR 
Reproducibility of Results/OR Discriminant Analysis/OR (psychometr* OR clinimetr* OR clinometr* OR (outcome ADJ3 (measure* OR assess*)) OR (observer* ADJ3 variation*) OR reproducib* OR reliab* OR unreliab* OR valid* OR coefficient OR homogen* OR "internal consistency" OR (cronbach* ADJ3 (alpha OR alphas)) OR (item* ADJ3 (correlation* OR selection* OR reduction*)) OR agreement OR precision OR imprecision OR "precise values" OR (test ADJ3 retest) OR (reliab* ADJ3 (test OR retest)) OR stability OR interrater OR intrarater OR ((intra OR inter) ADJ (rater OR tester OR observer OR technician OR examiner OR assay OR individual OR participant)) OR intertester OR intratester OR interobserver OR intraobserver OR intertechnician OR intratechnician OR interexaminer OR intraexaminer OR interassay OR intraassay OR interindividual OR intraindividual OR interparticipant OR intraparticipant OR kappa OR "kappa s" OR kappas OR repeatab* OR ((replicab* OR repeated) ADJ6 (measure OR measures OR findings $\mathrm{OR}$ result $\mathrm{OR}$ results $\mathrm{OR}$ test $\mathrm{OR}$ tests)) OR generali* OR concordance OR (intraclass ADJ3 correlation*) OR discriminative OR "known group" OR (factor ADJ (structure* OR analy*)) OR dimension* OR subscale* OR (multitrait AND (scaling ADJ3 analy*)) OR "item discriminant" OR (interscale ADJ correlation*) OR error OR errors OR ((individual OR interval OR rate) ADJ variability) $\mathrm{OR}$ (variability ADJ3 (analy* OR values)) $\mathrm{OR}$ (uncertainty $\mathrm{ADJ} 3$ (measurement $\mathrm{OR}$ measuring)) OR "standard error of measurement" OR sensitiv* OR responsive* OR (limit ADJ3 detection) OR "minimal detectable concentration" OR interpretab* OR ((minimal OR minimally OR clinical OR clinically) ADJ3 (important OR significant OR detectable) AND (change OR difference)) OR (small* ADJ3 (real OR detectable) AND (change OR difference)) OR "meaningful change" OR "ceiling effect" OR "floor effect" OR "Item response model" OR IRT OR Rasch OR "Differential item functioning" OR DIF OR “computer adaptive testing" OR "item bank" OR "cross-cultural equivalence").ab,ti.)

\section{References}

1. Stucki, G., Kostanjsek, N., Ustun, B., \& Cieza, A. (2008). ICFbased classification and measurement of functioning. European Journal of Physical and Rehabilitation Medicine, 44(3), 315-328.

2. Cieza, A., \& Stucki, G. (2008). The International Classification of Functioning Disability and Health: Its development process and content validity. European Journal of Physical and Rehabilitation Medicine, 44(3), 303-313.

3. Jelsma, J. (2009). Use of the International Classification of Functioning, Disability and Health: A literature survey. Journal of Rehabilitation Medicine, 41(1), 1-12.

4. Van Der Windt, D. A. W. M., Van Der Heijden, G. J. M. G., De Winter, A. F., Koes, B. W., Deville, W., \& Bouter, L. M. (1998).
The responsiveness of the Shoulder Disability Questionnaire. Annals of the Rheumatic Diseases, 57(2), 82-87.

5. Van Der Heijden, G. J. M. G., Leffers, P., \& Bouter, L. M. (2000). Shoulder disability questionnaire design and responsiveness of a functional status measure. Journal of Clinical Epidemiology, 53(1), 29-38.

6. Roach, K. E., Budiman-Mak, E., Songsiridej, N., \& Lertratanakul, Y. (1991). Development of a shoulder pain and disability index. Arthritis Care \& Research, 4(4), 143-149.

7. Hudak, P. L., Amadio, P. C., \& Bombardier, C. (1996). Development of an upper extremity outcome measure: The DASH (disabilities of the arm, shoulder, and head). American Journal of Industrial Medicine, 29(6), 602-608.

8. Bot, S. D., Terwee, C. B., van der Windt, D. A., Bouter, L. M., Dekker, J., \& de Vet, H. C. (2004). Clinimetric evaluation of shoulder disability questionnaires: A systematic review of the literature. Annals of the Rheumatic Diseases, 63(4), 335-341.

9. Roy, J. S., MacDermid, J. C., \& Woodhouse, L. J. (2009). Measuring shoulder function: A systematic review of four questionnaires. Arthritis and Rheumatism, 61(5), 623-632.

10. St-Pierre, C., Desmeules, F., Dionne, C. E., Fremont, P., MacDermid, J. C., \& Roy, J. S. (2016). Psychometric properties of self-reported questionnaires for the evaluation of symptoms and functional limitations in individuals with rotator cuff disorders: A systematic review. Disability and Rehabilitation, 38(2), 103-122. doi:10.3109/09638288.2015.1027004.

11. Angst, F., Schwyzer, H. K., Aeschlimann, A., Simmen, B. R., \& Goldhahn, J. (2011). Measures of adult shoulder function: Disabilities of the Arm, Shoulder, and Hand Questionnaire (DASH) and Its Short Version (QuickDASH), Shoulder Pain and Disability Index (SPADI), American Shoulder and Elbow Surgeons (ASES) Society Standardized Shoulder Assessment Form, Constant (Murley) Score (CS), Simple Shoulder Test (SST), Oxford Shoulder Score (OSS), Shoulder Disability Questionnaire. Arthritis Care \& Research, 63(SUPPL. 11), S174-S188.

12. Desai, A. S., Dramis, A., \& Hearnden, A. J. (2010). Critical appraisal of subjective outcome measures used in the assessment of shoulder disability. Annals of the Royal College of Surgeons of England, 92(1), 9-13.

13. Fayad, F., Mace, Y., \& Lefevre-Colau, M. M. (2005). Shoulder disability questionnaires: a systematic review. Annales de Réadaptation et de Médecine Physique, 48(6), 298-306.

14. Wright, R. W., Brand, R. A., Dunn, W., \& Spindler, K. P. (2007). How to write a systematic review. Clinical Orthopaedics and Related Research, 455, 23-29.

15. Beaton, D. E., Bombardier, C., Guillemin, F., \& Ferraz, M. B. (2000). Guidelines for the process of cross-cultural adaptation of self-report measures. Spine (Phila Pa 1976), 25(24), 3186-3191.

16. Wang, W. L., Lee, H. L., \& Fetzer, S. J. (2006). Challenges and strategies of instrument translation. Western Journal of Nursing Research, 28(3), 310-321.

17. Mokkink, L. B., Terwee, C. B., Knol, D. L., Stratford, P. W., Alonso, J., Patrick, D. L., et al. (2010). The COSMIN checklist for evaluating the methodological quality of studies on measurement properties: a clarification of its content. BMC Medical Research Methodology, 10, 22.

18. Mokkink, L. B., Terwee, C. B., Gibbons, E., Stratford, P. W., Alonso, J., Patrick, D. L., et al. (2010). Inter-rater agreement and reliability of the COSMIN (COnsensus-based Standards for the selection of health status Measurement Instruments) checklist. BMC Medical Research Methodology, 10, 82.

19. Schellingerhout, J. M., Heymans, M. W., Verhagen, A. P., de Vet, H. C., Koes, B. W., \& Terwee, C. B. (2011). Measurement properties of translated versions of neck-specific questionnaires: A systematic review. BMC Medical Research Methodology, 11, 87. 
20. Schellingerhout, J. M., Verhagen, A. P., Heymans, M. W., Koes, B. W., de Vet, H. C., \& Terwee, C. B. (2012). Measurement properties of disease-specific questionnaires in patients with neck pain: A systematic review. Quality of Life Research, 21(4), 659-670.

21. Mutsaers, J. H., Peters, R., Pool-Goudzwaard, A. L., Koes, B. W., \& Verhagen, A. P. (2012). Psychometric properties of the Pain Attitudes and Beliefs Scale for Physiotherapists: a systematic review. Man Ther, 17(3), 213-218.

22. van Bloemendaal, M., van de Water, A. T., \& van de Port, I. G. (2012). Walking tests for stroke survivors: A systematic review of their measurement properties. Disability and Rehabilitation, 34(26), 2207-2221. doi:10.3109/09638288.2012.680649.

23. Schellingerhout, J. M., Verhagen, A. P., Thomas, S., \& Koes, B. W. (2008). Lack of uniformity in diagnostic labeling of shoulder pain: time for a different approach. Manual Therapy, 13(6), 478-483.

24. Terwee, C. B., Jansma, E. P., Riphagen, I. I., \& de Vet, H. C. (2009). Development of a methodological PubMed search filter for finding studies on measurement properties of measurement instruments. Quality of Life Research, 18(8), 1115-1123.

25. Terwee, C. B., Mokkink, L. B., Knol, D. L., Ostelo, R. W., Bouter, L. M., \& de Vet, H. C. (2012). Rating the methodological quality in systematic reviews of studies on measurement properties: A scoring system for the COSMIN checklist. Quality of Life Research, 21(4), 651-657.

26. Nunally, J. C., \& Bernstein, I. H. (1994). Psychometric theory (3rd ed.). New York: McGraw-Hill.

27. Terwee, C. B., Bot, S. D., de Boer, M. R., van der Windt, D. A., Knol, D. L., Dekker, J., et al. (2007). Quality criteria were proposed for measurement properties of health status questionnaires. Journal of Clinical Epidemiology, 60(1), 34-42.

28. Mokkink, L. B., Terwee, C. B., Patrick, D. L., Alonso, J., Stratford, P. W., Knol, D. L., et al. (2010). The COSMIN study reached international consensus on taxonomy, terminology, and definitions of measurement properties for health-related patient-reported outcomes. Journal of Clinical Epidemiology, 63(7), 737-745.

29. van Tulder, M., Furlan, A., Bombardier, C., \& Bouter, L. (2003). Updated method guidelines for systematic reviews in the cochrane collaboration back review group. Spine (Phila $\mathrm{Pa}$ 1976), 28(12), 1290-1299.

30. Dogu, B., Sahin, F., Ozmaden, A., Yilmaz, F., \& Kuran, B. (2013). Which questionnaire is more effective for follow-up diagnosed subacromial impingement syndrome? A comparison of the responsiveness of SDQ, SPADI and WORC index. Journal of Back and Musculoskeletal Rehabilitation, 26(1), 1-7.

31. Paul, A., Lewis, M., Shadforth, M. F., Croft, P. R., Van Der Windt, D. A. W. M., \& Hay, E. M. (2004). A comparison of four shoulder-specific questionnaires in primary care. Annals of the Rheumatic Diseases, 63(10), 1293-1299.

32. Christiansen, D. H., Andersen, J. H., \& Haahr, J. P. (2013). Crosscultural adaption and measurement properties of the Danish version of the Shoulder Pain and Disability Index. Clinical Rehabilitation, 27(4), 355-360.

33. Ekeberg, O. M., Bautz-Holter, E., Keller, A., Tveita, E. K., Juel, N. G., \& Brox, J. I. (2010). A questionnaire found diseasespecific WORC index is not more responsive than SPADI and OSS in rotator cuff disease. Journal of Clinical Epidemiology, 63(5), 575-584.

34. Hill, C. L., Lester, S., Taylor, A. W., Shanahan, M. E., \& Gill, T. K. (2011). Factor structure and validity of the shoulder pain and disability index in a population-based study of people with shoulder symptoms. BMC Musculoskeletal Disorders, 12, 8.

35. Tveita, E. K., Sandvik, L., Ekeberg, O. M., Juel, N. G., \& BautzHolter, E. (2008). Factor structure of the Shoulder Pain and Disability Index in patients with adhesive capsulitis. BMC Musculoskeletal Disorders. doi:10.1186/1471-2474-9-103.
36. Tveita, E. K., Ekeberg, O. M., Juel, N. G., \& Bautz-Holter, E. (2008). Responsiveness of the shoulder pain and disability index in patients with adhesive capsulitis. BMC Musculoskeletal Disorders, 9, 161.

37. Ekeberg, O. M., Bautz-Holter, E., Tveita, E. K., Keller, A., Juel, N. G., \& Brox, J. I. (2008). Agreement, reliability and validity in 3 shoulder questionnaires in patients with rotator cuff disease. BMC Musculoskeletal Disorders, 9, 68.

38. Bicer, A., \& Ankarali, H. (2010). Shoulder Pain and Disability Index: A validation study in Turkish women. Singapore Medical Journal, 51(11), 865-870.

39. MacDermid, J. C., Solomon, P., \& Prkachin, K. (2006). The Shoulder Pain and Disability Index demonstrates factor, construct and longitudinal validity. BMC Musculoskeletal Disorders, 7, 12.

40. Staples, M. P., Forbes, A., Green, S., \& Buchbinder, R. (2010). Shoulder-specific disability measures showed acceptable construct validity and responsiveness. Journal of Clinical Epidemiology, 63(2), 163-170.

41. Jamnik, H., \& Spevak, M. K. (2008). Shoulder pain and disability Index: Validation of slovene version. International Journal of Rehabilitation Research, 31(4), 337-341.

42. Ozsahin, M., Akgun, K., Aktas, I., \& Kurtais, Y. (2008). Adaptation of the shoulder disability questionnaire to the Turkish population, its reliability and validity. International Journal of Rehabilitation Research, 31(3), 241-245.

43. de Winter, A. F., van der Heijden, G. J. M. G., Scholten, R. J. P. M., van der Windt, D. A. W. M., \& Bouter, L. M. (2007). The Shoulder Disability Questionnaire differentiated well between high and low disability levels in patients in primary care, in a cross-sectional study. Journal of Clinical Epidemiology, 60(11), 1156-1163.

44. Beaton, D. E., Katz, J. N., Fossel, A. H., Wright, J. G., Tarasuk, V., \& Bombardier, C. (2001). Measuring the whole or the parts? Validity, reliability, and responsiveness of the disabilities of the arm, shoulder and hand outcome measure in different regions of the upper extremity. Journal of Hand Therapy, 14(2), 128-146.

45. Haldorsen, B., Svege, I., Roe, Y., \& Bergland, A. (2014). Reliability and validity of the Norwegian version of the Disabilities of the Arm, Shoulder and Hand questionnaire in patients with shoulder impingement syndrome. BMC Musculoskeletal Disorders, 15(1), 78.

46. Offenbaecher, M., Ewert, T., Sangha, O., \& Stucki, G. (2002). Validation of a German version of the disabilities of arm, shoulder, and hand questionnaire (DASH-G). Journal of Rheumatology, 29(2), 401-402.

47. L'Insalata, J. C., Warren, R. F., Cohen, B. A. S. B., Altchek, D. W., \& Peterson, M. G. E. (1997). A self-administered questionnaire for assessment of symptoms and function of the shoulder. Journal of Bone and Joint Surgery Series A, 79(5), 738-748.

48. Vermeulen, H. M., Boonman, D. C. G., Schuller, H. M., Obermann, W. R., van Houwelingen, H. C., Rozing, P. M., \& Vliet Vlieland, T. P. M. (2005). Translation, adaptation and validation of the Shoulder Rating Questionnaire (SRQ) into the Dutch language. Clin Rehabil, 19(3), 300-311.

49. Lippitt, S. B., Harryman, D. T., \& Matsen, F. A. (1993). A practical tool for evaluating function: the Simple Shoulder Test. In The shoulder: a balance of mobility and stability (pp. 501-518). Rosemont: The American Academy of Orthopaedic Surgeons.

50. van Kampen, D. A., van Beers, L. W. A. H., Scholtes, V. A. B., Terwee, C. B., \& Willems, W. J. (2012). Validation of the Dutch version of the Simple Shoulder Test. Journal of Shoulder and Elbow Surgery, 21(6), 808-814.

51. Tashjian, R. Z., Deloach, J., Green, A., Porucznik, C. A., \& Powell, A. P. (2010). Minimal clinically important differences in ASES and simple shoulder test scores after nonoperative treatment of rotator cuff disease. Journal of Bone and Joint Surgery American, 92(2), 296-303. 
52. Mintken, P. E., Glynn, P., \& Cleland, J. A. (2009). Psychometric properties of the shortened disabilities of the Arm, Shoulder, and Hand Questionnaire (QuickDASH) and Numeric Pain Rating Scale in patients with shoulder pain. Journal of Shoulder and Elbow Surgery, 18(6), 920-926.

53. Godfrey, J., Hamman, R., Lowenstein, S., Briggs, K., \& Kocher, M. (2007). Reliability, validity, and responsiveness of the simple shoulder test: Psychometric properties by age and injury type. Journal of Shoulder and Elbow Surgery, 16(3), 260-267.

54. Croft, P., Pope, D., Zonca, M., O’Neill, T., \& Silman, A. (1994). Measurement of shoulder related disability: Results of a validation study. Annals of the Rheumatic Diseases, 53(8), 525-528.

55. Terwee, C. B., Schellingerhout, J. M., Verhagen, A. P., Koes, B. W., \& de Vet, H. C. (2011). Methodological quality of studies on the measurement properties of neck pain and disability questionnaires: a systematic review. Journal of Manipulative and Physiological Therapeutics, 34(4), 261-272.

56. Wild, D., Eremenco, S., Mear, I., Martin, M., Houchin, C., Gawlicki, M., et al. (2009). Multinational trials-recommendations on the translations required, approaches to using the same language in different countries, and the approaches to support pooling the data: The ISPOR patient-reported outcomes translation and linguistic validation good research practices task force report. Value Health, 12(4), 430-440.

57. Reeve, B. B., Wyrwich, K. W., Wu, A. W., Velikova, G., Terwee, C. B., Snyder, C. F., et al. (2013). ISOQOL recommends minimum standards for patient-reported outcome measures used in patient-centered outcomes and comparative effectiveness research. Quality of Life Research, 22(8), 1889-1905.

58. Beaton, D. E., Wright, J. G., \& Katz, J. N. (2005). Development of the QuickDASH: comparison of three item-reduction approaches. Journal of Bone and Joint Surgery. American Volume, 87(5), 1038-1046.

59. Beaton, D. E., Wright, J. G., \& Katz, J. N. (2005). Development of the QuickDASH: Comparison of three item-reduction approaches. Journal of Bone and Joint Surgery American, 87A(5), 1038-1046.
60. Turner, R. R., Quittner, A. L., Parasuraman, B. M., Kallich, J. D., Cleeland, C. S., \& Mayo, F. D. A. P.-R. O. C. M. G. (2007). Patient-reported outcomes: Instrument development and selection issues. Value Health, 10(Suppl 2), S86-S93.

61. Rose, M., Bjorner, J. B., Becker, J., Fries, J. F., \& Ware, J. E. (2008). Evaluation of a preliminary physical function item bank supported the expected advantages of the Patient-Reported Outcomes Measurement Information System (PROMIS). Journal of Clinical Epidemiology, 61(1), 17-33.

62. Hays, R. D., Spritzer, K. L., Amtmann, D., Lai, J. S., Dewitt, E. M., Rothrock, N., et al. (2013). Upper-extremity and mobility subdomains from the Patient-Reported Outcomes Measurement Information System (PROMIS) adult physical functioning item bank. Archives of Physical Medicine and Rehabilitation, 94(11), 2291-2296.

63. Cloke, D. J., Lynn, S. E., Watson, H., Steen, I. N., Purdy, S., \& Williams, J. R. (2005). A comparison of functional, patient-based scores in subacromial impingement. Journal of Shoulder and Elbow Surgery, 14(4), 380-384.

64. Fan, Z. J., Smith, C. K., \& Silverstein, B. A. (2008). Assessing validity of the QuickDASH and SF-12 as surveillance tools among workers with neck or upper extremity musculoskeletal disorders. Journal of Hand Therapy, 21(4), 354-365.

65. Offenbacher, M., Ewert, T., Sangha, O., \& Stucki, G. (2003). Validation of a German version of the 'Disabilities of Arm, Shoulder and Hand' questionnaire (DASH-G). Zeitschrift fur Rheumatologie, 62(2), 168-177.

66. Alvarez-Nemegyei, J., Puerto-Ceballos, I., Guzman-Hau, W., Bassol-Perea, A., \& Nuno-Gutierrez, B. L. (2005). Development of a Spanish-language version of the Shoulder Disability Questionnaire. J Clin Rheumatol, 11(4), 185-187.

67. Jeldi, A. J., Aseer, A. L., Dhandapani, A. G., \& Roach, K. E. (2012). Cross-cultural adaption, reliability and validity of an Indian (Tamil) version for the Shoulder Pain and Disability Index. Hong Kong Physiother J, 30(2), 99-104. 